\title{
Inverse spin Hall effect induced by asymmetric illumination of light in topological insulator $\mathrm{Bi}_{2} \mathrm{Se}_{3}$
}

\author{
Di Fan ${ }^{\circ},{ }^{*}$ Rei Hobara, Ryota Akiyama, ${ }^{\dagger}$ and Shuji Hasegawa \\ Department of Physics, The University of Tokyo, Tokyo 113-0033, Japan
}

(Received 11 October 2019; accepted 6 March 2020; published 20 April 2020)

\begin{abstract}
Illumination of circularly polarized light is an alternative to electrical methods for spin injection. Spins are injected at the point of light illumination, and then they diffuse radially due to the in-plane gradient of the spin density. This diffusing spin current is converted into a circular charge current by the inverse spin Hall effect (ISHE) and is usually not detectable. However, by shining a light on an asymmetric part of the sample, such as near the edges, we detected this current as a helicity-dependent component in the measured photocurrent. This is not due to the circular photogalvanic effect because it occurs even at normal incidence of the light. A finite-element-method simulation shows that the ISHE-induced circular charge current generates an electric dipole at the edge of the sample, causing the measured charge current. The asymmetric light illumination shown here is a simple way to control the output of the ISHE, which may lead to novel spintronic devices.
\end{abstract}

DOI: 10.1103/PhysRevResearch.2.023055

\section{INTRODUCTION}

Spin injection and detection are the two key factors for spintronics. So far, most researchers have been using strong spin-orbit coupling (SOC) materials to detect in-plane spin injected from a ferromagnetic (FM) layer. To convert spin into a charge current, we can rely on the inverse spin Hall effect (ISHE) [1,2] or the spin-momentum locking effect on the Fermi surface, the so called inverse Rashba-Edelstein effect (IREE) $[3,4]$. In addition to conventional electrical spin injection, many attempts have been made to use laser light to control electron spins $[5,6]$. One such example is the circular photogalvanic effect (CPGE), which is an optical way to convert the angular momentum of light into the orbital angular momentum (OAM) of electron states. Since the coupling between spin and OAM exists in SOC materials, a spindependent charge current occurs. This has been demonstrated in various materials [7-11].

The idea of a photoinduced ISHE (PISHE) was demonstrated in an experiment as early as the 1980s [12]. Bakunn et al. used a circularly polarized light to inject out-of-plane spin in bulk Ge. They observed a charge current generated by the ISHE under an external magnetic field, which is used to align the spin of photoexcited carriers. Recently, some groups reported that the PISHE was realized in two-dimensional electron gas (2DEG) with strong SOC [13] and topological insulators [14] without magnetic fields.

In the present research, we propose a simple way to simultaneously inject spin and detect the ISHE, that is, laser

\footnotetext{
*fan@surface.phys.s.u-tokyo.ac.jp

†akiyama@surface.phys.s.u-tokyo.ac.jp
}

Published by the American Physical Society under the terms of the Creative Commons Attribution 4.0 International license. Further distribution of this work must maintain attribution to the author(s) and the published article's title, journal citation, and DOI. illumination on an edge of a topological insulator $\mathrm{Bi}_{2} \mathrm{Se}_{3}$ thin film. Compared with previous studies, our method shows higher ISHE conversion efficiency, due to the cutoff of the charge current loop at the sample edge. In addition, it is easier to detect the ISHE in such an experimental scheme. Although high laser power density was required to observe a clear PISHE in previous studies [13,14], it could lead to many side effects, such as sample damages, significant thermoelectric effects, and nonlinear optical effects, since $\mathrm{Bi}_{2} \mathrm{Se}_{3}$ is known as a thermoelectric material with high nonlinear optical susceptibility [15]. Our method of using a low-power laser is a good way to avoid or reduce these side effects. In addition, we also present a FEM simulation based on a new model, which indicates that this effect comes from the breaking of the circular charge current due to the sample edge and shows a virtual electric dipole. Such a model agrees well with experimental results. These suggest that the PISHE could possibly be observed on any strong SOC material with such asymmetric illumination. When normal incidence of light is applied to inject the out-of-plane spin, the resultant spin current is converted to a circular charge current by the ISHE and finally to the one-way charge current

\section{BACKGROUND}

When injecting spin into a material, the diffusing spin current $J_{d}$ would be generated following the diffusion equation,

$$
J_{d}=D \cdot \nabla N .
$$

Here, $D$ and $N$ are the diffusion constant and the spin density, respectively. If the ISHE occurs in this situation, the induced charge current $J_{\text {ISHE }}$ can be written as

$$
J_{\text {ISHE }}=\theta_{k} J_{d} \times S_{z},
$$

where $\theta_{k}$ and $S_{z}$ are the spin Hall angle and the projection of the spin in the $z$ direction (surface normal), respectively. The $z$-direction spin could originate from the bulk excitation, or 


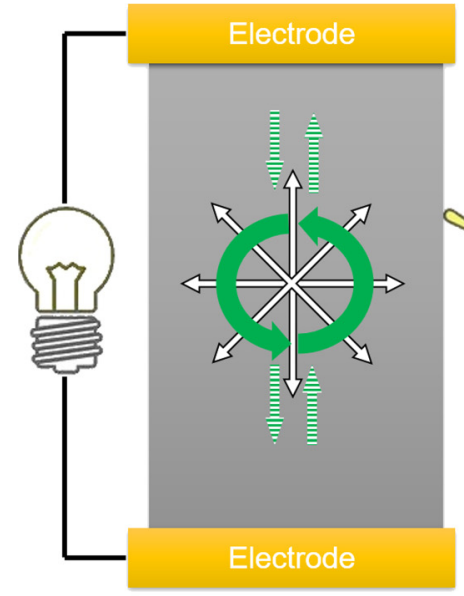

(a)

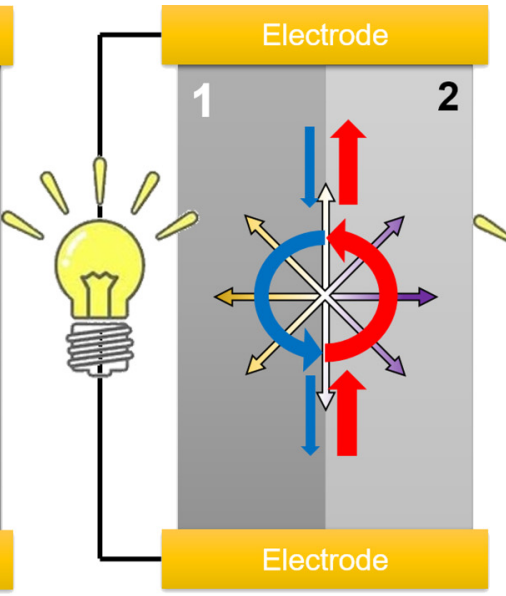

(b)

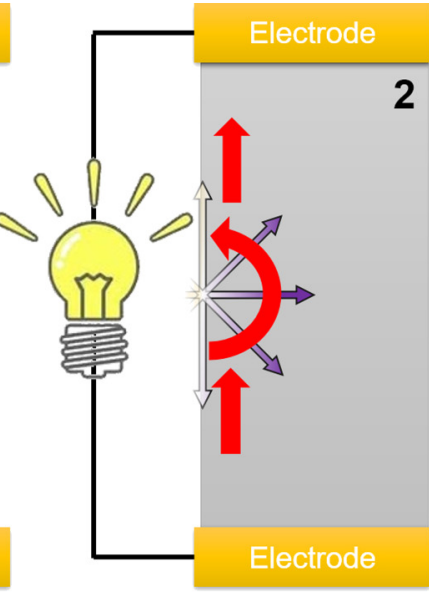

(c)

FIG. 1. (a) When the circularly polarized light irradiates the center on the sample surface, the circular charge current (green solid arrows), which is converted from the radial spin current (white solid arrows) by the ISHE, appears. However, no outcome can be detected by the electrodes at both ends of the sample. The dashed green arrows are the inflow and outflow of the closed circular electric current, canceling each other, resulting in no net flow along the sample. (b) When the circularly polarized light irradiates an interface between different materials, 1 and 2, the inflow and outflow currents are not equivalent, resulting in a net current flowing along the longitudinal direction. (c) As one of the simplest situations of (b), when the material 1 is vacuum, the net current is expected to be the maximum.

from the surface bands with the $z$-direction spin component. As for the $z$-direction spin component in the surface bands, previous studies show that the hexagonal warping effect appears at the topological surface states and the 2DEG Rashba spin splitting states of $\mathrm{Bi}_{2} \mathrm{Se}_{3}[16,17]$, which leads to an outof-plane spin polarization at the vicinity of the Fermi surface.

When the spins are injected at a point of light illumination, the injected spins diffuse and spread out radially on the sample surface. Such a radial diffusion of the injected spins will, according to Eq. (2), yield a circular charge current when the spins are in the $z$ direction. However, the electric current observed between the electrodes at both ends of the sample is zero, when the light is irradiated at the center of the sample, because the induced charge current is "canceled out," as shown in Fig. 1(a).

On the other hand, when the laser light illuminates the interface between two different materials, where the carrier diffusion in Material 1 is smaller than that in Material 2, as shown in Fig. 1(b), the charge currents induced by the ISHE are not equivalent between the two parts, and a net charge current flowing in one direction would appear. For the simplest and most practical case, we can assume that Material 1 is vacuum, and Material 2 is the sample to be investigated. Obviously, there is no diffusion current in the vacuum side, thus the circular charge current is expected to be detected by the electrodes at both ends of the sample as shown in Fig. 1(c).

Following this scheme, we injected out-of-plane spin into a thin film and detected the charge current induced by the ISHE. The experiments were performed by illuminating circularly polarized light at normal incidence near the edge of the sample, which is the topological insulator $\mathrm{Bi}_{2} \mathrm{Se}_{3}$ thin film grown on a $\mathrm{Si}$ substrate. In addition, we show a control experiment with a bare $\mathrm{Si}$ substrate that is a weak SOC material. As expected, the ISHE was detected at the edge of the strong SOC thin film but not presented at all on the weak SOC material.
Previously, the CPGE was observed on $\mathrm{Bi}_{2} \mathrm{Se}_{3}$ flakes using lasers with the wavelength $\lambda$ of 780 and $1064 \mathrm{~nm}[18,19]$, which showed the conversion of the in-plane spin to the charge current since the light was illuminated with off-normal incidence. In our experiments, the laser with $\lambda=1550 \mathrm{~nm}$ was

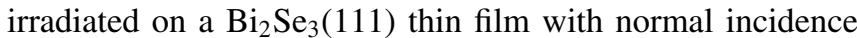
of light to inject the out-of-plane spin into the film. The film was 13 quintuple-layer (QL) thick, grown by molecular beam epitaxy (MBE) technique on a $3 \mathrm{~mm} \times 8 \mathrm{~mm}$ $\mathrm{Si}(111)$ substrate [20]. The sample was capped with $\mathrm{Al}_{2} \mathrm{O}_{3}$ before being transferred into the measurement chamber. The sample was clamped mechanically at both ends by tungsten electrodes. More information about the sample can be found in Appendixes A and B. Since the photon energy of $0.8 \mathrm{eV}$ $(1550 \mathrm{~nm})$ is smaller than the band-gap energy of the $\mathrm{Si}$ substrate $(1.12 \mathrm{eV})$, the observed photocurrent originates from the $\mathrm{Bi}_{2} \mathrm{Se}_{3}$ film only. During the experiment, the laser power was kept as low as $1.75 \mathrm{~mW}$, which was confirmed not to damage the film. All measurements with laser illumination were performed in an ultrahigh-vacuum chamber at room temperature without removing the capping layer.

\section{EXPERIMENTAL RESULTS}

First, we examined the incident-angle dependence together with the irradiation-position dependence as shown in Fig. 2. The photocurrent $J$ detected by the electrodes at both ends is related with the polarization of the light, and thus should depend on the rotation angle $\phi$ of the quarter-wave plate (QWP) in the light path as

$$
J=C \sin 2 \phi+L_{1} \sin 4 \phi+L_{2} \cos 4 \phi+D .
$$

Here, by changing $\phi$, the light polarization is modulated among linearly $\left(\phi=0^{\circ}, 90^{\circ}\right)$ polarized and right-handed $\left(\phi=45^{\circ}\right) /$ left-handed $\left(\phi=135^{\circ}\right)$ circularly polarized light. 


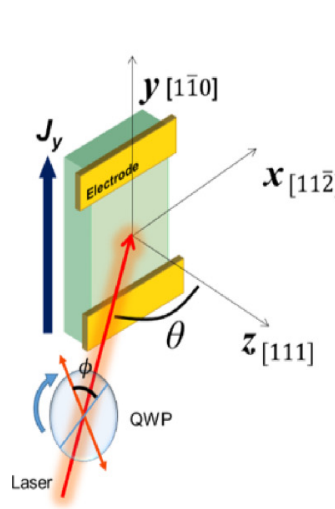

(a)

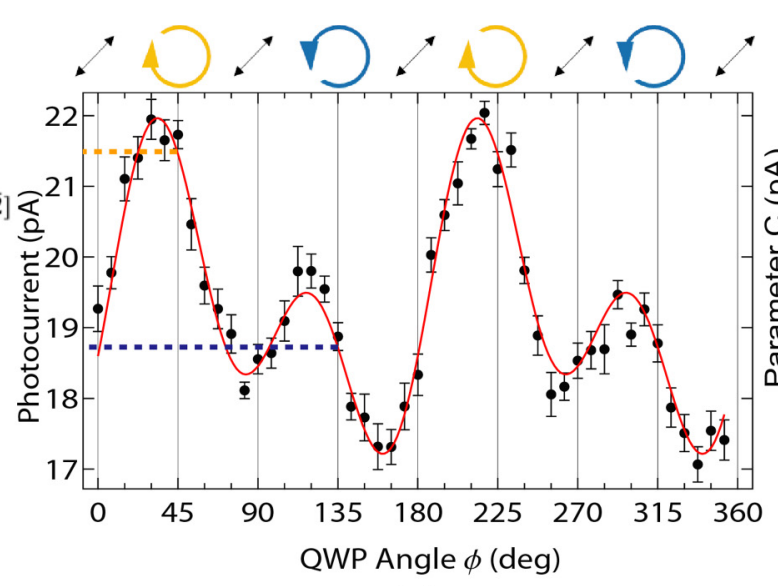

(b)

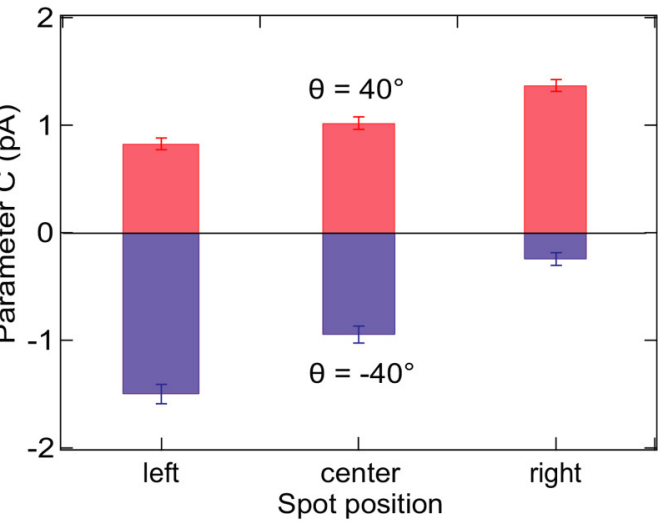

(c)

FIG. 2. (a) Experimental coordinate based on a silicon substrate. (b) Polarization-dependent photocurrent taken by irradiating laser light near the edge of the sample under incident angle $\theta=40^{\circ}$, and the curve was fitted by Eq. (3). (c) Spot position (near left and right edges and the center of the sample) dependence of the parameter $C$ estimated from the fitting at the incident angle $\theta$ of $40^{\circ}$ and $-40^{\circ}$ with respect to the surface-normal direction. The sign of parameter $C$ is reversed when the incident angle is reversed, but the position dependence is in the same trend for both incident angles. Note that parameter $C$ in this case includes contributions both from the CPGE and the ISHE, and the latter has the negative contribution at the left edge and the positive contribution at the right edge in this system, irrespective of the sign of the incidence angle of light. At the center of the sample, only the CPGE contributes to $C$.

The most interesting and important helicity-dependent photocurrent (HDP) is represented by the fitting factor $C$.

As for the CPGE of $\mathrm{Bi}_{2} \mathrm{Se}_{3}$, the crystal symmetry $\left(C_{3 v}\right)$ of a $\mathrm{Bi}_{2} \mathrm{Se}_{3}(111)$ thin film forbids the CPGE at normal incidence of light. Therefore, to observe the CPGE, the oblique light is irradiated, and in-plane spin is injected. Then, due to the spinmomentum locking effect, the induced charge current flows in one direction $[8,18]$.

When the incident plane of the light is set in the $x z$-plane, the CPGE current along the $y$-direction on the surface of a $\mathrm{Bi}_{2} \mathrm{Se}_{3}(111)$ thin film is expected as shown in Fig. 2(a), and it is written as [22]

$$
\mathrm{j}_{y}^{\mathrm{CPGE}}=-\gamma_{x y} \hat{e}_{x} P_{\text {circ }} E_{0}^{2},
$$

where $P_{\text {circ }}$ is the degree of circular polarization, $E_{0}$ is the electric field amplitude, and $\hat{e}_{x}$ is the $x$ projection of the unit vector pointing in the direction of the light propagation $\left(\hat{e}_{x}=\right.$ $\sin \theta$, where $\theta$ is the angle between the light direction and the surface-normal direction). Then, the CPGE is expected to disappear under normal incidence, i.e., incident along $z$-direction, because $\hat{e}_{x}$ is zero.

Here we can see that the parameter $C$ for the incidence angle of $\pm 40^{\circ}$ has opposite signs, as shown in Fig. 2(c), which is exactly the nature of the CPGE; as shown in Eq. (1), the CPGE photocurrent reverses its sign when the incident direction (in-plane component $\hat{e}_{x}$ ) is reversed. On the other hand, the distinct position dependence, as shown in Fig. 2(c), infers a different mechanism other than the CPGE. As shown in Fig. 2(c), the tendency of the position dependence does not change despite the incident angle changes, which suggests that the position dependence is not related with the in-plane component of the angular momentum of the light. It is strongly suggested that the out-of-plane component can be attributed to the edge behavior.

To confirm this hypothesis, the photocurrent at normal incidence was measured. Intriguingly, the HDP was nonzero even at normal incidence, especially when the light spot was near the edge of the sample. This cannot be explained by the CPGE mechanism reported in previous studies $[8,21,22]$ as the crystal symmetry $\left(C_{3 v}\right)$ of $\mathrm{Bi}_{2} \mathrm{Se}_{3}(111)$ thin film forbids the CPGE at normal incidence of light.

Though the irradiation partially on the side surface of the $\mathrm{Bi}_{2} \mathrm{Se}_{3}$ film is possible and the edge of the sample may break the symmetry to enable the CPGE, this CPGE should be extremely small, as explained in Appendix C.

The results can be understood as follows: the circularly polarized light excites electrons and holes with the out-of-plane spin. The electrons and holes diffuse in the same directions along each concentration gradient. The diffusion of carriers with spins is converted into a charge current by the ISHE. Therefore, the parameter $C$ represents the ISHE component dominantly.

The parameters $L_{1}$ and $L_{2}$ indicate the photocurrent generated by the linearly polarized light. Specifically, $L_{1}$ represents the linear photogalvanic effect (LPGE), which arises in a crystal without an inversion center, and it is related to the crystalline symmetry [12]. However, $L_{2}$ is beyond the expectation of the LPGE, and some reports explain it as the anomalous linear photogalvanic effect (ALPGE) [23]. $D$ represents an offset current that is not related to the polarization of the light, and it may originate from the photo Dember effect and/or the thermal effect.

Figure 3 shows the QWP-angle dependences of the photocurrent when the laser light illuminates the right edge, the center, and the left edge of the sample, respectively. The amplitudes of the photocurrent at the left and right edges are significantly larger than that at the center. Moreover, the HDP, indicated by the difference between the anticlockwise (orange) and clockwise (blue dashed lines) circularly polarized light, is opposite between the left- and right-edge irradiations, whereas it is negligible for the center irradiation. As a control experiment on a bare silicon substrate [ $\mathrm{Si}(111)$ 


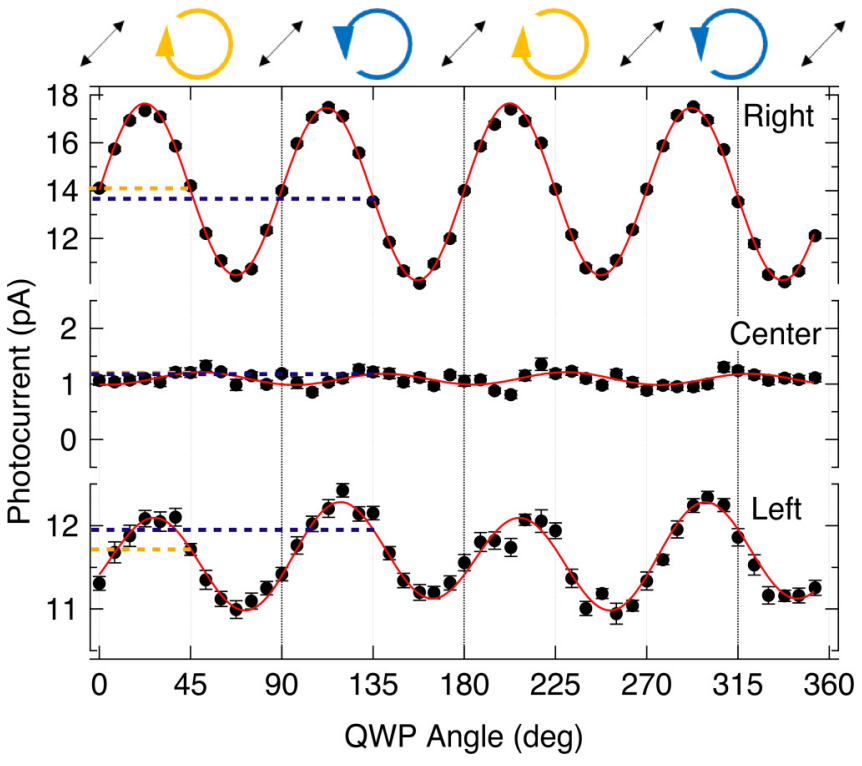

FIG. 3. Polarization dependences of the photocurrent measured at normal incidence of light. Polarization was changed by rotating the QWP. The points were the average of 20 times scan raw data. Red lines are fitting curves by Eq. (3). The orange (blue) dashed line indicates the photocurrent at the right-handed (left-handed) circular polarization. The difference between the two dashed lines corresponds to HDP, twice the magnitude of parameter $C$.

also has $C_{3 v}$ symmetry] is shown in Fig. 9 in Appendix D, the amplitude of the photocurrent takes a maximum at the center of the sample, while it decreases at the edge of the sample, which is normal since part of the light is outside of the sample. Moreover, there is no HDP at any of the positions.

To investigate further, we look into the position dependence of fitting parameters after fitting the data with Eq. (3). The illumination position dependences of the fitting parameter $C$ and the others are shown in Figs. 4(a) and 4(b), respectively. The prominent difference between these parameters is that the parameter $C$ changes antisymmetrically with respect to the sample center, whereas the other factors are symmetric. When the laser spot (diameter $\sim 1.2 \mathrm{~mm}$ ) was focused on the sample surface, the charge current induced by the ISHE was enhanced at both edges of the sample while almost zero at the center, as indicated by red circles in Fig. 4(a). Remarkably, the sign of the parameter $C$ is opposite on opposite sides of the sample, meaning that the HDP flows on the right and left edges in the directions opposite to each other, as expected in the scheme of Fig. 1(c). If this result originates from a thermal effect or any other effects by a laser hotspot, one would expect the position dependence to be symmetric with respect to the sample center. For more discussion on thermal effects, please refer to Appendix D.

Figure 5 shows the results of the finite-element method (FEM) simulation to investigate the potential distribution on the sample induced by the spin injection. Equations used for the calculation of ISHE are described by Eqs. (10)-(13) in Ref. [24]. Injected spin distribution follows a step function as $I=\left\{\begin{array}{l}1, x \leqslant r \\ 0, x>r\end{array}\right.$. As shown in Fig. 5(b), when the spin injection takes place at the center of the sample, no electric potential is generated. On the other hand, as in Fig. 5(a), when the spins are injected on the left edge of the sample, remarkably, a finite electric potential due to an electric dipole is created by the ISHE. The dipole center is the spin injection point, and

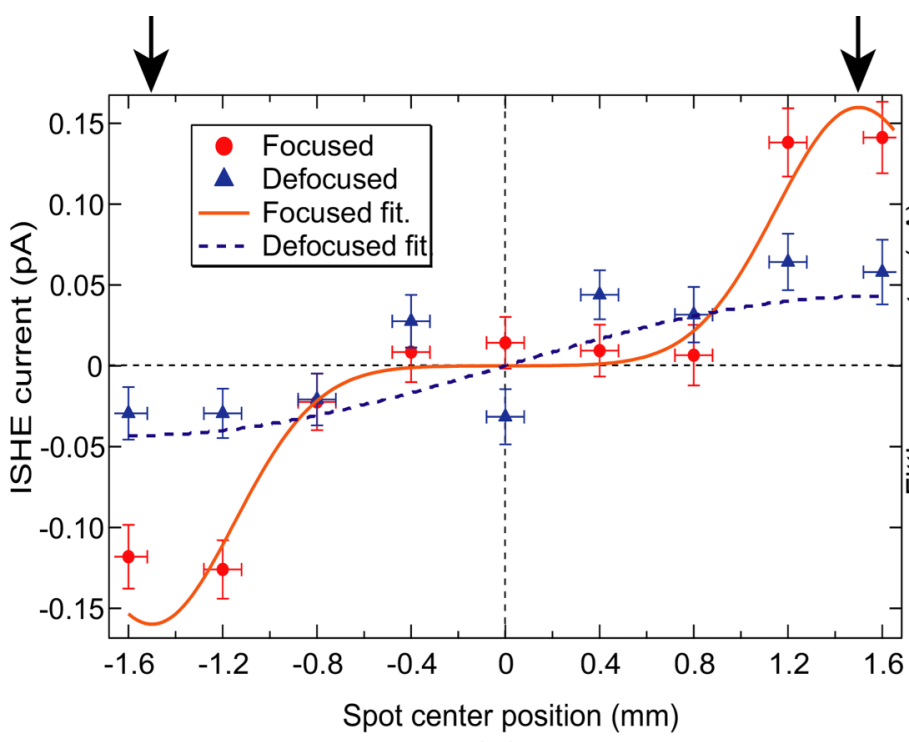

(a)

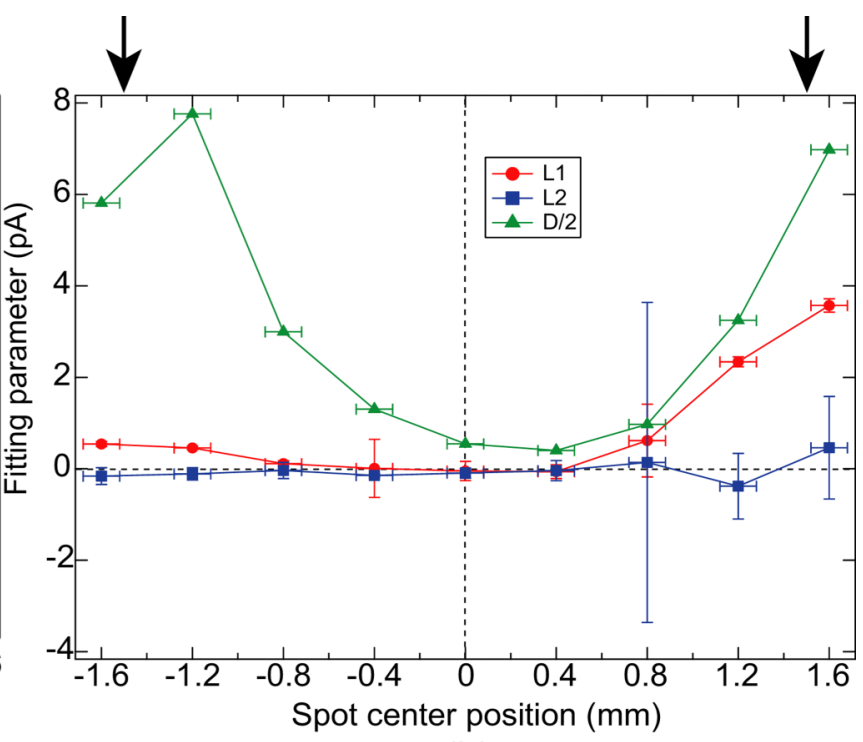

(b)

FIG. 4. (a) Dependence of the parameter $C$ on the laser spot position across the sample, with the focused (red circles) and defocused (blue triangles) laser at normal incidence, while keeping the same average power. The positions of sample edges are indicated by black arrows at $\pm 1.5 \mathrm{~mm}$. The radius with the focused and defocused laser spot is estimated to be $\sim 0.6$ and $\sim 1.2 \mathrm{~mm}$, respectively, by fittings (see Appendix E). The curves are the calculated results from Eqs. (E13) and (E14) for the respective spot sizes. (b) Position dependence of the parameters $L_{1}, L_{2}$, and $D / 2$ of the focused laser. Non-antisymmetric shapes are seen, which might originate from usual edge effects such as the thermal effect or an effect caused by the laser hotspot. 


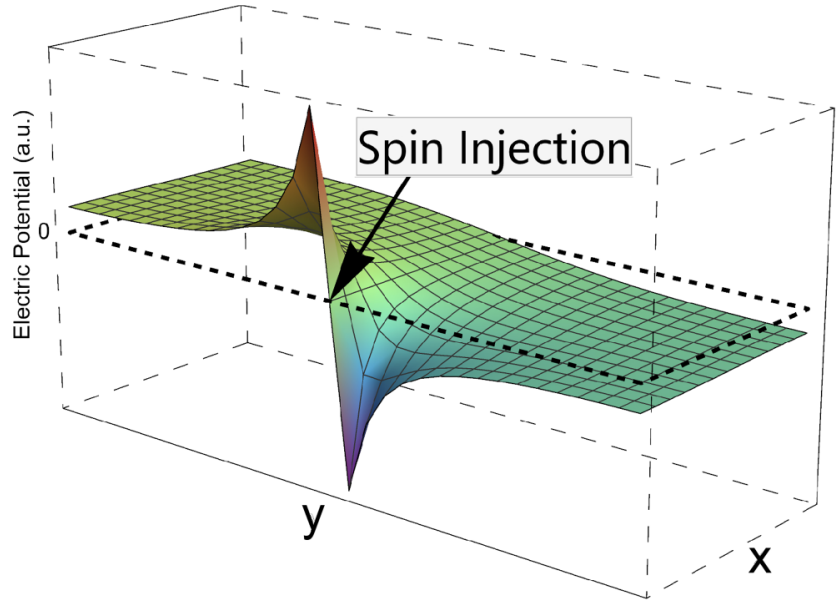

(a)

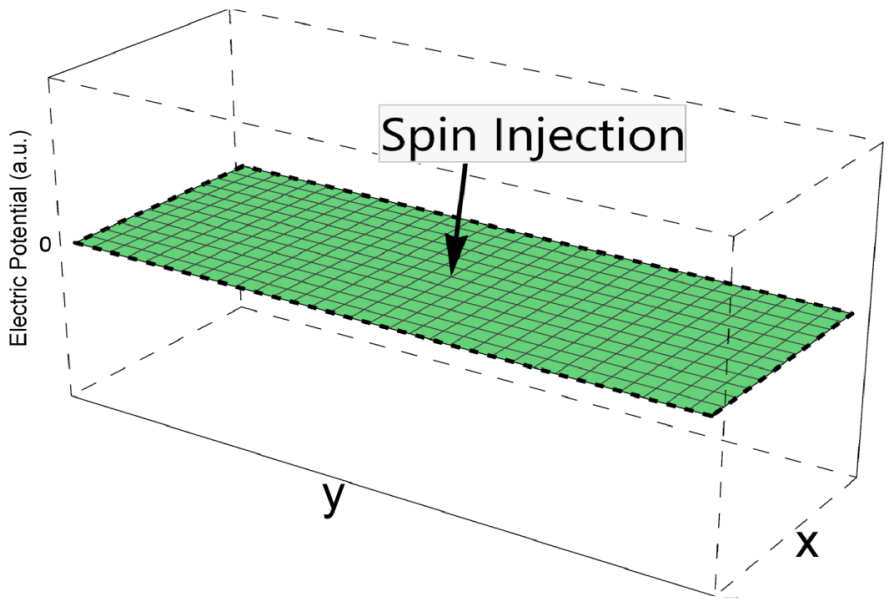

(b)

FIG. 5. FEM simulations of the electric potential distribution at equilibrium after out-of-plane spin is injected. The $x$ - and $y$-axes are positions on the sample, whereas the electric potential in the vertical axis is in arbitrary units. The arrows point to the spin injection positions. The dashed thick line indicates the neutral potential position. (a) A situation in which the spin injection occurs at the left edge of the sample. Two peaks are seen with opposite signs, which represent generation of an electric dipole at the edge. (b) A situation in which spins are injected at the center of the sample. No potential difference is generated.

the two peaks of the positive and negative electric potential are at the edge of the sample. Similarly, when spins are injected at the right edge, a dipole but with opposite sign is created.

The HDP, shown by the parameter $C$, observed at normal incidence of light, and the potential distribution in the FEM simulation originate from the same origin, namely the ISHE. It can be interpreted that the circular charge current path induced by the ISHE acts like a conveyer; carriers are transferred from one part of the edge to the other along the half-circular trajectory. Consequently, an electric potential builds up due to the carrier accumulation. When the field of this electric dipole is balanced by the ISHE, no more carriers are accumulated and the system is in equilibrium, resulting in a potential difference along the $y$ direction, yielding the measured HDP.

\section{DISCUSSION}

During the optical excitation, electron-hole pairs are generated by the circular polarized light, and these carriers diffuse outward radially from the spot center. Due to the energy and angular momentum conservations at the optical excitation, the electron and hole gain the angular momentum of a circularly polarized photon of $+\hbar$ or $-\hbar$. The electrons and holes with the same spin diffuse outward radially from the spot center, which is a pure spin current without a charge current. Usually the diffusion coefficient of electrons is larger than that of holes, thus electrons diffuse down faster along the gradient of density, leading to a density imbalance between electrons and holes, which creates an electric field. This electric field acts to decelerate electrons and accelerate holes until both diffusions reach the same rate. In this equilibrium, there is no net charge current radially along the electrons/holes diffusion direction (so-called ambipolar diffusion [25]). If the carrier lifetime is long enough to fulfill this equilibrium, a pure spin current is created by photoexcitation. Then, due to the
ISHE, the electrons and holes with the same spin will flow in opposite directions along the half-circular path, leading to charge current being detected, which is enhanced compared with the case of electron flow only. Moreover, the chargecurrent direction along the edge (the polarity of the dipole) changes depending on up or down of the injected spin, which is also opposite between the right and left edges of the sample.

The difference of the electric potential between the two electrodes should strongly depend on the spatial distribution of the injected spin and the distance between the injection point and the sample edge. In this experiment, these are represented by laser spot profiles and the distance between the spot center and the sample edge. In our system, the spin diffusion length is much smaller than the spot size. Hence, the region where the spin current flows is determined by the spot size. Consequently, the dipole distance is restrained by the spot size, and the dipole appears only when the circular spot is partially cut by the edge of the sample. That is the reason why the parameter $C$ is nonzero only at a limited area near the edge, as shown in Fig. 4(a).

Recently, we have been made aware of a similar experiment reported in $\mathrm{Bi}_{2} \mathrm{Te}_{2} \mathrm{Se}$ [26]. Seifert et al. discovered this zero-bias HDP (shown in their supplemental materials) at the edges of their sample, and they think that the CPGE of the side surface may be the origin of this HDP. By comparing our results, we suggest that their findings may have the same origin as ours, that is, the inverse spin Hall effect due to symmetry breaking at the sample edges.

\section{CONCLUSION}

In conclusion, we observed the HDP due to the ISHE

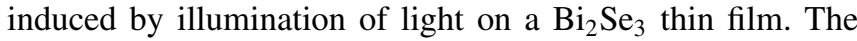
detection of out-of-plane spins is well demonstrated by experiments and supported by FEM simulations. It suggests that an electric dipole is generated at the edge of the sample, resulting 


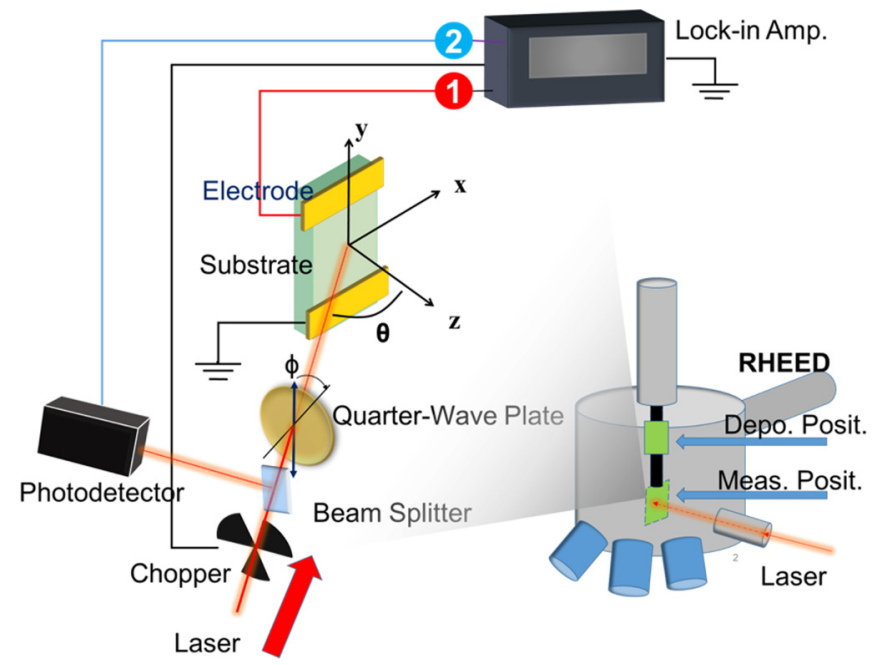

FIG. 6. Experimental setup of sample growth and optical measurements.

from the accumulation of carriers steered by the ISHE. This scheme for detection of a spin current will pave the way for novel spintronics devices operating at room temperature without an external magnetic field, and it will help to make spin-charge conversion more accessible.

\section{ACKNOWLEDGMENTS}

We acknowledge financial supports from the Japan Society of Promotion of Science (KAKENHI JP16H02108, 25110010, 25246025, 22246006, JP16H00983, JP15K21717) and MEXT (Grant-in-Aid for Scientific Research on Innovative Areas "Molecular Architectonics" 25110001). D.F. also likes to thank for the financial support from The Advanced Leading Graduate Course for Photon Science (ALPS) of the MEXT "Program for Leading Graduate Schools."

\section{APPENDIX A: EXPERIMENTAL SETUP}

The experimental apparatus is shown in Fig. 6. An IR laser $(1550 \mathrm{~nm})$ was aligned with the help of a visible $(635 \mathrm{~nm})$ laser in the setup. The spot-center positions of the two laser beams at the cofocus point were separated within $1 \mathrm{~mm}$. During measurements, the visible laser was blocked, and a chopper was operated at $293 \mathrm{~Hz}$. The whole optical stage could be moved along the $x$ direction by a homemade mechanical facility. Due to the configuration restriction in our apparatus, the laser spot is not exactly at the center of the sample in the $y$ direction; it is at 3-4 $\mathrm{mm}$ above the lower electrode. The $\mathrm{Si}(111)$ substrate was $3 \mathrm{~mm} \times 8 \mathrm{~mm} \times 0.5 \mathrm{~mm}$ in size.

\section{APPENDIX B: SAMPLE PREPARATION}

The thin film of $\mathrm{Bi}_{2} \mathrm{Se}_{3}$ was grown on a $\mathrm{Si}(111)$ substrate in an ultrahigh-vacuum (UHV) chamber (Fig. 6) by the molecular-beam-epitaxy technique. First we prepared the $\mathrm{Si}(111)-\beta-\sqrt{ } 3 \times \sqrt{ } 3$-Bi surface structure by depositing one atomic layer of $\mathrm{Bi}$, and then proceeded to $\mathrm{Bi}_{2} \mathrm{Se}_{3}$ growth. The flux ratio of $\mathrm{Bi}$ to $\mathrm{Se}$ was between 1:10 and 1:20. The substrate was kept at $\sim 175{ }^{\circ} \mathrm{C}$ during the growth of the first $\mathrm{QL} \mathrm{Bi} \mathrm{Se}_{3}$, and after that the substrate temperature was raised and kept at $\sim 200{ }^{\circ} \mathrm{C}$ for further growth. The growth rate was $\sim 4 \mathrm{~min} / \mathrm{QL}$. The diffraction pattern of $\mathrm{Bi}_{2} \mathrm{Se}_{3}$ is displayed in Fig. 7(a), and the thickness was monitored by the reflection-high-energy electron diffraction (RHEED) intensity oscillation in situ as shown in Fig. 7(b). It is known that $n$-type $\mathrm{Bi}_{2} \mathrm{Se}_{3}$ is usually formed under such a growth procedure due to Se vacancies in the film.

From the previous angle-resolved photoemission spectroscopy (ARPES) reports, in addition to Dirac-cone-type topological surface states, Rashba-type spin-split conduction bands of the film also exist at the Fermi surface of $\mathrm{Bi}_{2} \mathrm{Se}_{3}$ [16]. The hexagonal warping at the Rashba-type states and topological surface states $[16,27]$ in the vicinity of the Fermi surface is known to play an important role for the out-of-plane spin component.

For ex situ measurement, a $\sim 2 \mathrm{ML}$ Al layer was deposited on the $\mathrm{Bi}_{2} \mathrm{Se}_{3}$ thin film. Then, the sample was taken out of the UHV chamber, resulting in immediate formation of the $\mathrm{Al}_{2} \mathrm{O}_{3}$ capping layer by oxidation [28].

\section{APPENDIX C: CIRCULAR PHOTOGALVANIC EFFECT}

The circular photogalvanic effect (CPGE) is related with the crystal symmetry of the sample. The symmetry group of the $\mathrm{Bi}_{2} \mathrm{Se}_{3}$ crystal is $D_{3 d}$, which includes the inversion symmetry. Generally, the photogalvanic effect occurs only in the crystal structure without inversion symmetry. Therefore, for $\mathrm{Bi}_{2} \mathrm{Se}_{3}$, the CPGE is allowed only at the surface of $\mathrm{Bi}_{2} \mathrm{Se}_{3}$ where the inversion symmetry in the $z$-direction is broken and the symmetry at the surface is reduced to $C_{3 v}$. The second rank pseudotensor for $C_{3 v}$ symmetry is

$$
\left(\begin{array}{ccc}
0 & \gamma_{x y} & 0 \\
-\gamma_{x y} & 0 & 0 \\
0 & 0 & 0
\end{array}\right) .
$$

Following Eq. (4) in the body of the article, photocurrent can be expected in the $y$ direction when light is irradiated obliquely in the $x z$ plane on the sample. As for our main discussion, one may argue that the symmetry would be different at the edge of the sample, thus the CPGE could be possible at the edge even if the measurement is performed at normal incidence $\left(\hat{e}_{x}=0\right)$. When threefold symmetry is broken at the edges, mirror symmetry may survive at the flat edge regions (the mirror plane is perpendicular to the edge line). In this case, the second-rank pseudotensor is

$$
\left(\begin{array}{ccc}
0 & \gamma_{x y} & 0 \\
\gamma_{y x} & 0 & \gamma_{z y} \\
0 & \gamma_{y z} & 0
\end{array}\right) .
$$

Due to the nonzero $\gamma_{y z}$ value, the CPGE can be observed along the $y$ direction even at normal incidence following the CPGE equation, $j_{\lambda}^{\mathrm{CPGE}}=\gamma_{\lambda \mu} \hat{e}_{\mu} P_{\text {circ }} E_{0}^{2}$ in Ref. [22]. However, the sign of the CPGE should be the same on both edges, which is obviously different from what we have observed. As one can learn from the above equation, photocurrent along the $y$-direction obtained from the CPGE will not change its direction unless the laser propagating direction $\hat{e}_{\mu}$ is reversed. Therefore, even if the edge shows other symmetries that can 


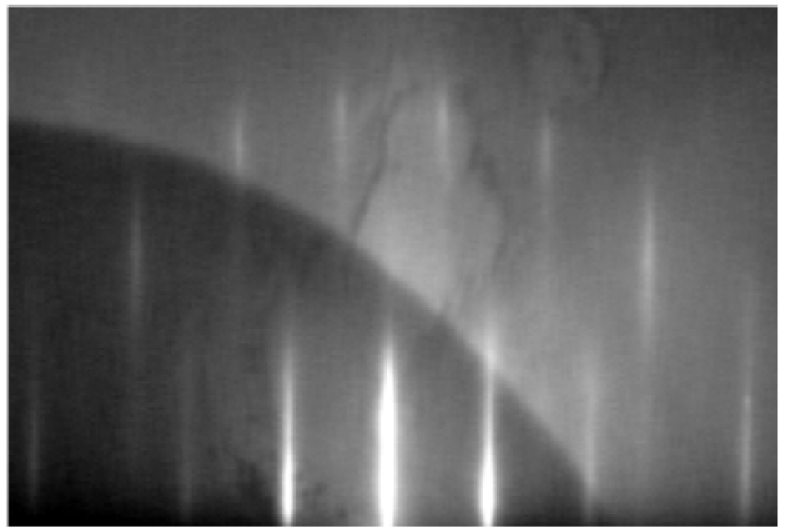

(a)

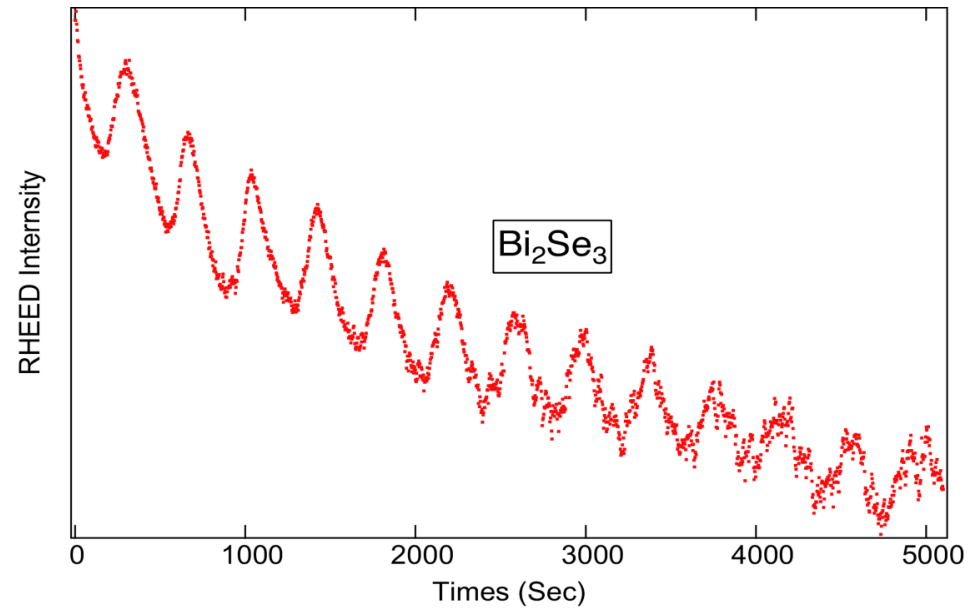

(b)

FIG. 7. (a) RHEED pattern taken after the growth of $\mathrm{Bi}_{2} \mathrm{Se}_{3}$ film. (b) Intensity oscillation of the specular spot in RHEED observed during the $\mathrm{Bi}_{2} \mathrm{Se}_{3}$ growth.

induce the CPGE, the sign of the CPGE would be the same on both edges of the sample.

In addition, the side surface of the thin film can also generate the CPGE photocurrent, and in this case the opposite side surface generates the opposite-direction CPGE photocurrent. However, this CPGE photocurrent is so small for our thin-film sample.

For example, we measured the CPGE of a $13 \mathrm{QL} \mathrm{Bi}_{2} \mathrm{Se}_{3}$ at $\theta=40^{\circ}$ in Fig. 2, schematic as Fig. 8, and the signal reads around $1 \mathrm{pA}$ with a full-scale laser beam (diameter $=1.2 \mathrm{~mm}$ ). The thickness of a 13-QL-thick $\mathrm{Bi}_{2} \mathrm{Se}_{3}$ film is $13 \mathrm{~nm}$, therefore the side surface is $13 \mathrm{~nm}$ wide. Compared with the full-scale beam diameter under the oblique incidence, the CPGE from the side surface should be $13 \mathrm{~nm} / 1.2 \mathrm{~mm}$ $10^{-5} \mathrm{pA}$. On the other hand, as shown in Fig. 2, when the laser illuminates at the sample center with $\theta=40^{\circ}$, the HDP is fully originated from the CPGE, which is $\sim 1 \mathrm{pA}$. As seen in Fig. 2(c), the magnitude of HDP is $\sim 1 \mathrm{pA}+(-) 0.2 \mathrm{pA}$ at the left- (right-) side edge. This $0.2 \mathrm{pA}$ does not come from the CPGE due to symmetry breaking because it is much larger than the estimated value above. Therefore, the observed HDP at the sample edges is not dominated by the CPGE.

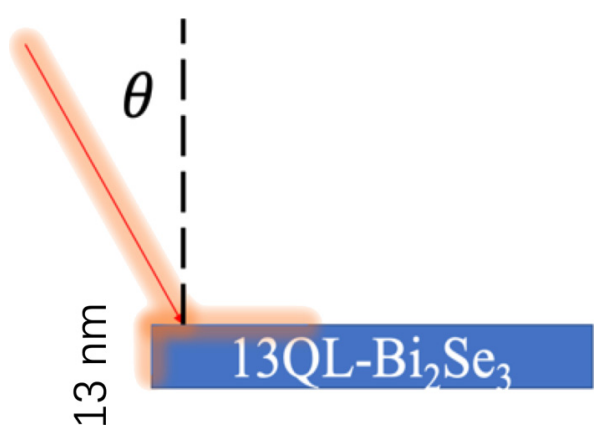

FIG. 8. The schematic picture of the edge illumination of a $13 \mathrm{QL}-\mathrm{Bi}_{2} \mathrm{Se}_{3}$.

\section{APPENDIX D: THERMAL INFLUENCES}

Besides the nature of a topological insulator, $\mathrm{Bi}_{2} \mathrm{Se}_{3}$ is also a well-known thermoelectric material. Therefore, thermal effects induced by a laser needed to be considered carefully. While the laser is moving along the transverse $(x)$ direction, the temperature gradient in the transversal direction may change. This change in the transverse direction has an impact on both the transverse and longitudinal $(y)$ directions. For the transverse direction, a thermal current would flow but cannot be measured by our setup. For the longitudinal direction, a spin potential could be built by the spin Nernst effect, which is, however, not measurable by our setup. In addition, an electrical potential in the longitudinal direction generated by the anomalous Nernst effect [29] will be included in the measurement results.

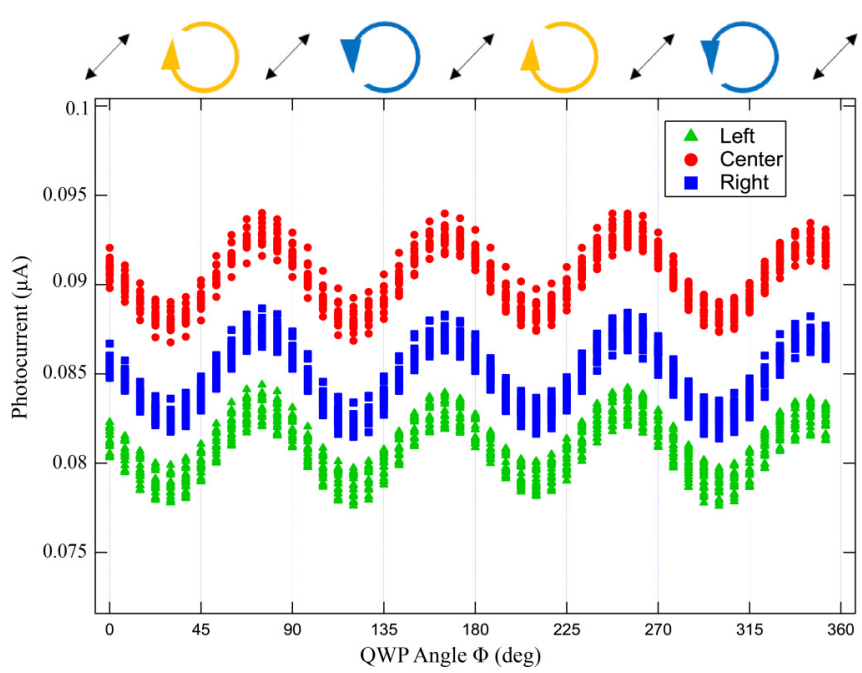

FIG. 9. A control experiment on a bare $\mathrm{Si}(111)$ substrate by laser of $\lambda=635 \mathrm{~nm}$. Photocurrent observed at the left edge and the right edge is almost the same as the center. No HDP was observed, either. 
Due to the strong spin-orbit coupling, the Nernst effect without an external magnetic field, i.e., the anomalous Nernst effect, converts the transverse thermal gradient into a longitudinal electrical potential difference. However, this potential difference is not spin-dependent, which means this potential should not depend on the polarization of the laser light. Our fitting result in Fig. 4(b) shows that the fitting parameter $D$ is not exactly symmetric with respect to the center of the sample; this could be the influence of the anomalous Nernst effect.

Here we show a $0.5 \mathrm{~mW}, 635 \mathrm{~nm}$ laser normally incident on different positions on a bare $\mathrm{Si}(111)$ substrate as shown in Fig. 9. The photocurrent shows the maximum at the center of the sample, and no helicity-dependent photocurrent was observed; the right-handed and left-handed circularly polarized light produces the same photocurrent at all places.

\section{APPENDIX E: SYMMETRY BREAKING ISHE INDUCED BY THE CIRCULARLY POLARIZED LIGHT}

As is known, the laser irradiance distribution in the spot is a Gaussian. Since a Gaussian wave propagates along the $z$-axis (normal to the sample surface), the irradiance at a distance $r$ on the sample plane ( $x y$ ) from the center of spot is expressed as

$$
\begin{gathered}
I\left(r, z_{0}\right)=I_{0} \exp \left(\frac{-r^{2}}{2 \sigma^{2}}\right), \\
(\mathrm{E} 1) \quad \begin{array}{l}
\text { the } G(x, y, \theta) \text { in Eq. (E4) to obtain the diffusion } \\
\text { at }(x, y) \text { in vector form, and } \boldsymbol{J}_{d} \text { is given as }
\end{array} \\
\boldsymbol{J}_{d}=-A D\left\{\frac{\left(x-x_{\mathrm{o}}\right) \cos ^{3} \theta}{2 \sigma^{4} \pi} \exp \left(-\frac{\left(x-x_{\mathrm{o}}\right)^{2} \cos ^{2} \theta+y^{2}}{2 \sigma^{2}}\right), \frac{y \cos \theta}{2 \sigma^{4} \pi} \exp \left(-\frac{\left(x-x_{\mathrm{o}}\right)^{2}+-y^{2}}{2 \sigma^{2}}\right)\right\} .
\end{gathered}
$$

where $2 \sigma$ indicates the radius of the laser spot $\left(1 / e^{2}\right.$ definition). The spot center is at $\left(x_{0}, y_{0}\right)$ on the $x y$ plane, and the incident angle $\theta$ with respect to the surface-normal direction is in the $x z$-plane. The intensity distribution $I$ is given as

$$
\begin{gathered}
I=I_{0} G(x, y, \theta), \\
G(x, y, \theta)=\frac{\cos \theta}{2 \sigma^{2} \pi} \exp \left(-\frac{\left(x-x_{\mathrm{o}}\right)^{2} \cos ^{2} \theta+\left(y-y_{0}\right)^{2}}{2 \sigma^{2}}\right) .
\end{gathered}
$$

This directly influences the density distribution of the photoexcited carriers $N(x, y)$. Moreover, the carrier distribution generates a diffusing spin current $\boldsymbol{J}_{d}$ along the concentration gradient. According to the diffusion equation,

$$
J_{d}=D \cdot \nabla N \text {. }
$$

Here the concentration of photoexcited carriers $N$ should be proportional to the irradiance of the light. Therefore, it is represented by the same Gaussian distribution $G(x, y, \theta)$. $D$ is the diffusion coefficient for the ambipolar diffusion of photoexcited carriers (electrons and holes). For simplicity, the spot center is at $\left(x_{0}, 0\right)$ on the $x$-axis. Then we can substitute the $G(x, y, \theta)$ in Eq. (E4) to obtain the diffusion spin current
Here, $A$ is a coefficient related to the excitation probability of carriers by the light. Equation (E5) means that the spin current flows out radially from the spot center.

Due to the ISHE, this spin current generates charge current $J^{\mathrm{ISHE}}$ as

$$
J^{\mathrm{ISHE}}=\theta_{k} \cdot \boldsymbol{J}_{d} \times \boldsymbol{S}_{z},
$$

where $\theta_{\mathrm{k}}$ is the spin Hall angle and $\boldsymbol{S}_{z}$ is the $z$-component of spin of the photoexcited carriers. The direction of the charge current is always perpendicular to the direction of the spin current. Then, since the spin current flows radially from the laser spot center, the charge current flows circularly around the laser spot. In Fig. 10(b), the diffusion spin current (red arrows) and charge current induced by the ISHE (yellow arrows) are plotted together with irradiance of the laser (background color gradation). To get rid of the CPGE and to focus on the ISHE only, we set $\theta$ to be zero, which is the case of normal incidence.

As we suggested in the main article, the system goes to equilibrium and there is no charge transfer from one part to another at the edge; the ISHE current at the edge is balanced by the electric field due to the dipole. The Poisson equation for the whole area of the sample gives the potential distribution $\varphi(x, y)$ when the laser spot center is at the left edge of the

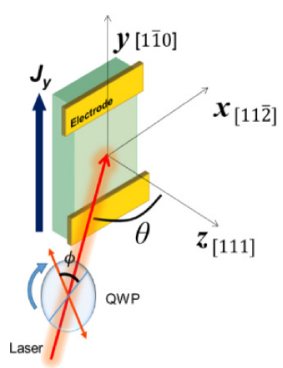

(a)

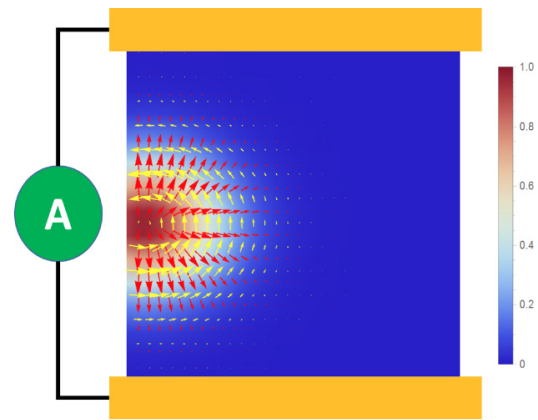

(b)
FIG. 10. (a) Schematic of the experimental setup. The coordinate based on a silicon substrate is shown; $x$-axis along [112], $y$-axis along [110], and $z$-axis along [111] directions, respectively. The $x z$ plane is the plane of incidence of light. The incident angle $\theta$ is the angle between the laser propagation direction and the $z$-axis. By rotating the quarter-wave plate (QWP), the polarization of the initially linearly polarized laser is changed. The photocurrent $J_{y}$ was measured along the $y$-axis by the electrodes at both ends. (b) A simulation result of the ISHE. The color gradient shows the distribution of the laser intensity, and thus it indicates the distribution of the photoexcited carrier density (and also the spin density). Red arrows show the diffusion direction of carriers and spin, and yellow arrows indicate the charge current induced by the ISHE. Arrow lengths indicate the intensities of respective currents. 


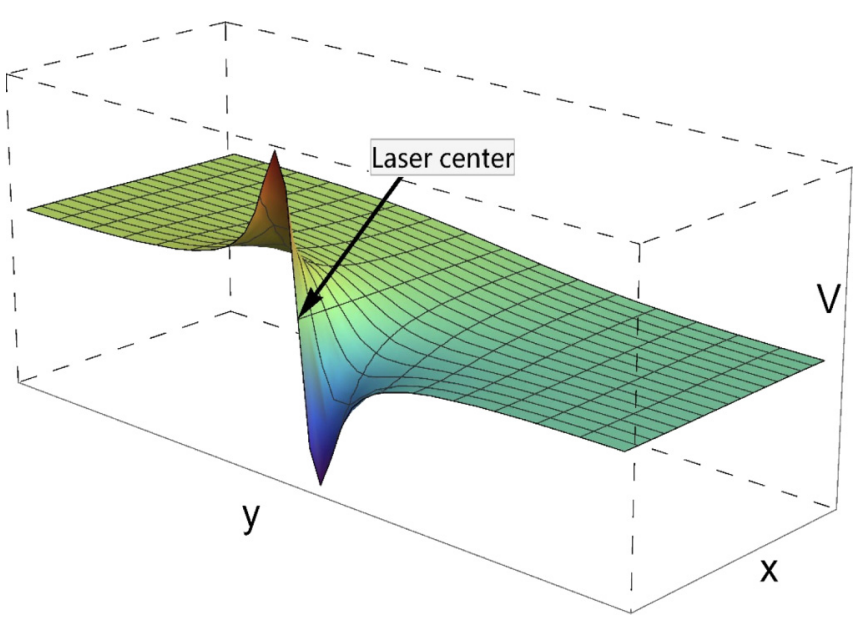

FIG. 11. By solving the Poisson equation (E7) with the boundary conditions (E8) and (E9), the electric potential distribution can be calculated. The numerical solution is plotted as a color plot, and it is similar to the potential distribution calculated by the FEM from Eqs. (10) to (13) in Ref. [21].

sample as

$$
\begin{gathered}
\nabla \cdot\left[\sigma_{1} \text { or } 2 \nabla \varphi(x, y)\right]=0, \\
\left.\sigma_{2} \boldsymbol{n} \cdot \nabla \varphi(x, y)\right|_{x=-w}=-J_{x}^{\mathrm{ISHE}}(-w, y), \\
\varphi(x, 0)=0 .
\end{gathered}
$$

Note that $\boldsymbol{n}$ is the unit vector perpendicular to the left edge of the sample, and $\sigma_{1}$ and $\sigma_{2}$ are the conductance of the metallic electrodes and sample, respectively. $w$ is half of the sample width, and thus the left edge is $x=-w$. Equation (E9) is assumed by the symmetry of the system. From Eqs. (E7)-(E9), the numerical solution for the potential distribution on the sample surface was obtained as shown in Fig. 11. This result shows almost the same distribution as the FEM calculation [Fig. 5(a)] in the main text, which is derived from Eqs. (10)-(13) in Ref. [21].

It is difficult to obtain the analytical solution of the equations above, thus we tried another way to describe the electric potential of our system. Here, we assume that the charges exist only on the sample edge without electrodes (i.e., along the $y$-direction in Fig. 10), and the charge density is proportional to the amplitude of the ISHE current. When the dipole distance is small enough, the dipole electric potential can be

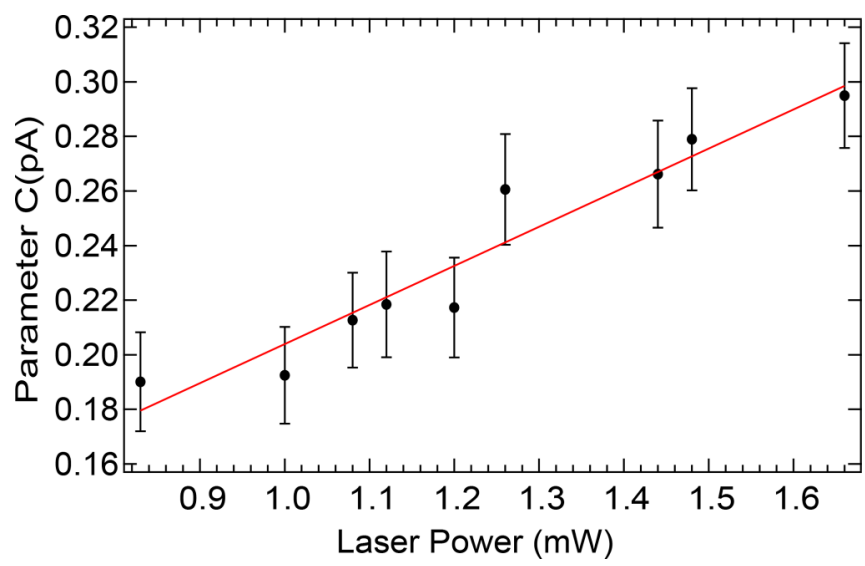

FIG. 12. Laser power dependence of the ISHE current (parameter $C$ ) at normal incidence. The laser spot was focused to the minimum size, and the spot position was near the sample edge to maximize the ISHE.

expressed as

$$
\varphi(r)=\frac{k p \cos \beta}{r^{2}}
$$

where $k$ is Coulomb's constant, $\boldsymbol{p}=q \boldsymbol{d}$ is defined as the dipole moment ( $\boldsymbol{d}$ is the displacement vector pointing from the negative charge $-q$ to the positive charge $+q$ in the dipole), $\beta$ is the angle between $\boldsymbol{r}$ and $\boldsymbol{p}$, and $r$ is the distance from the dipole center. As mentioned above, for the $J^{\mathrm{ISHE}}$ at both edges of the sample $(w, y)$ or $(-w, y), \boldsymbol{p}$ should be

$$
\boldsymbol{p}=J_{x= \pm w}^{\mathrm{ISHE}} y d y
$$

and then the voltage between the two electrodes at both ends of the sample is

$$
V=2 k \int_{0}^{l} \frac{J_{x=w}^{\mathrm{ISHE}} y d y \cos \theta}{r^{2}}+2 k \int_{0}^{l} \frac{J_{x=-w}^{\mathrm{ISHE}} y d y \cos \theta}{r^{2}} \text {. }
$$

Here, $l$ is the half-length of the sample along the $y$-direction. The potential distribution along the $x$-direction at the same $y$ is similar, assuming that the potential at equilibrium is just smoothed from the potential at the same $y$. Thus, the final expression for the voltage between the electrodes at both ends is

$$
V=k A D \frac{e^{-\frac{2 w+2 x_{0}{ }^{2}+l^{2}}{2 \sigma^{2}}}\left(e^{\frac{\left(w-x_{0}\right)^{2}}{2 \sigma^{2}}}-e^{\frac{\left(w+x_{0}\right)^{2}}{2 \sigma^{2}}}\right)\left(2 l-\sigma e^{\frac{l^{2}}{2 \sigma^{2}}} \sqrt{2 \pi} \operatorname{erf}\left(\frac{l}{\sigma \sqrt{2}}\right)\right)}{4 \sigma^{2} \pi n^{2}} .
$$

$\left(x_{\mathrm{o}}, 0\right)$ is the spot position $\left(x_{\mathrm{o}} \in[-w, w]\right)$. By knowing the resistance of the sample $R$, the measured current $J_{y}$ flowing between the electrodes is

$$
J_{y}=\frac{V}{R} .
$$

From Eq. (E13), we are able to investigate the ISHE further. First, as we assumed earlier, the ISHE current should be linearly proportional to the light intensity as experimentally observed (Fig. 12). Moreover, we can use Eq. (E13) to fit the position dependence as in Fig. 3 of the main article. 
[1] E. Saitoh et al., Appl. Phys. Lett. 88, 182509 (2006).

[2] T. Kimura, Y. Otani, T. Sato, S. Takahashi, and S. Maekawa, Phys. Rev. Lett. 98, 156601 (2007).

[3] J. C. Rojas-Sánchez, L. Vila, G. Desfonds, S. Gambarelli, J. P. Attané, J. M. De Teresa, C. Magén, and A. Fert, Nat. Commun. 4, 2944 (2013).

[4] J. C. Rojas-Sánchez et al., Phys. Rev. Lett. 116, 096602 (2016).

[5] F. Meier and B. P. Zakharchenya, Optical Orientation (Elsevier, Amsterdam, 2012).

[6] S. Pfalz, R. Winkler, T. Nowitzki, D. Reuter, A. D. Wieck, D. Hägele, and M. Oestreich, Phys. Rev. B 71, 165305 (2005).

[7] S. D. Ganichev, E. L. Ivchenko, S. N. Danilov, J. Eroms, W. Wegscheider, D. Weiss, and W. Prettl, Phys. Rev. Lett. 86, 4358 (2001).

[8] K. N. Okada, N. Ogawa, R. Yoshimi, A. Tsukazaki, K. S. Takahashi, M. Kawasaki, and Y. Tokura, Phys. Rev. B 93, 081403(R) (2016).

[9] N. Ogawa, M. S. Bahramy, Y. Kaneko, and Y. Tokura, Phys. Rev. B 90, 125122 (2014).

[10] H. Yuan et al., Nat. Nanotech. 9, 851 (2014).

[11] S. Dhara et al., Science 349, 726 (2015).

[12] A. A. Bakun et al., JETP Lett. 40, 1293 (1984).

[13] X. W. He et al., Phys. Rev. Lett. 101, 147402 (2008).

[14] J. Yu et al., Nano Lett. 17, 7878 (2017).
[15] J. W. McIver, D. Hsieh, S. G. Drapcho, D. H. Torchinsky, D. R. Gardner, Y. S. Lee, and N. Gedik, Phys. Rev. B 86, 035327 (2012).

[16] P. D. C. King, R. C. Hatch, M. Bianchi, R. Ovsyannikov, C. Lupulescu, G. Landolt, B. Slomski, J. H. Dil, D. Guan et al., Phys. Rev. Lett. 107, 096802 (2011).

[17] Y. H. Wang, D. Hsieh, D. Pilon, L. Fu, D. R. Gardner, Y. S. Lee, and N. Gedik, Phys. Rev. Lett. 107, 207602 (2011).

[18] J. W. McIver et al., Nat. Nanotech. 7, 96 (2012).

[19] J. Duan et al., Sci. Rep. 4, 4889 (2014).

[20] Y. Sakamoto, T. Hirahara, H. Miyazaki, S. I. Kimura, and S. Hasegawa, Phys. Rev. B 81, 165432 (2010).

[21] Y. Q. Huang et al., Nat. Commun. 8, 15401 (2017).

[22] S. D. Ganichev et al., Physica E 14, 166 (2002).

[23] X. Y. Peng et al., Phys. Rev. B 84, 075341 (2011).

[24] R. V. Shchelushkin and A. Brataas, Phys. Rev. B 71, 045123 (2005).

[25] N. Kumar, B. A. Ruzicka, N. P. Butch, P. Syers, K. Kirshenbaum, J. Paglione, and H. Zhao, Phys. Rev. B 83, 235306 (2011).

[26] P. Seifert et al., Nat. Commun. 9, 331 (2018).

[27] T. Hirahara, Y. Sakamoto, Y. Takeichi, H. Miyazaki, S. I. Kimura, I. Matsuda, A. Kakizaki, and S. Hasegawa, Phys. Rev. B 82, 155309 (2010).

[28] M. Lang et al., ACS Nano 6, 295 (2011).

[29] P. Sengupta and J. Shi, Phys. Rev. Mater. 2, 064606 (2018). 